\title{
Dynamic resource allocation for cognitive radio based smart grid communication networks
}

\author{
Sheraz Alam ${ }^{1,2, *}$, Mubashar Sarfraz ${ }^{1}$, M. B. Usman ${ }^{1}$, M. A. Ahmad ${ }^{1}$, Shareena Iftikhar ${ }^{1}$ \\ ${ }^{1}$ Department of Engineering, National University of Modern Languages, Islamabad, Pakistan \\ 2 Department of Electronics Engineering, International Islamic University, Islamabad, Pakistan
}

\section{A R T I C L E I N F O}

\section{Article history:}

Received 31 May 2017

Received in revised form

15 August 2017

Accepted 23 August 2017

\section{Keywords:}

Cognitive radio

Smart grid communication network

Fairness

Max sum reward

\begin{abstract}
A B S T R A C T
Cognitive Radio (CR) is a new technology to answer the spectrum shortage problem by dynamically allowing secondary (unlicensed) users to utilize the spectrum holes, avoiding interference with primary (licensed) users. Smart Grid (SG) is an enhancement of the conventional system of electricity distribution and management. Two-way communication, smart devices, and sensors are the core competencies of SG which result in increasing the efficiency and reliability of the SG system. An enormous amount of data in the range of thousands of Terabytes is expected to be generated due to various SG applications in a fully functional smart grid communication network (SGCN), requiring a fair share of spectrum resources. CR based SGCN is widely proposed in the literature to carry a major chunk of this data to increase spectral efficiency. Dynamic spectrum allocation on the basis of fairness using CR technology is proposed in this work, which ensures the fair distribution of spectrum resources among cognitive SG users. This optimization problem is solved using heuristic approach. A comparative analysis of three algorithms: genetic algorithm (GA), particle swarm optimization (PSO) and cat swarm optimization (CSO) is presented for evaluating fairness and max sum reward (MSR). It is shown that CSO outperforms both GA and PSO in terms of average fairness and MSR achieved by secondary users for a number of allocations.
\end{abstract}

(C) 2017 The Authors. Published by IASE. This is an open access article under the CC BY-NC-ND license (http://creativecommons.org/licenses/by-nc-nd/4.0/).

\section{Introduction}

In the last few years, the demand for wireless communication is increasing day by day and the available spectrum resources are becoming scarce. According to the FCC's report, unreasonable command and control spectrum regulation is the main cause of underutilization of the wireless spectrum (FCC, 2002). The available electromagnetic spectrum cannot be increased and we are left with the only solution that we fully utilize the available spectrum resources.

Static spectrum allocation is causing a huge waste of available spectrum with low consumption rate. Dynamic Spectrum Access (DSA) (Brown, 2005) can be the best option to overcome this wastage. Cognitive radio (CR), a promising technology to improve spectral efficiency is proposed in this work to solve the problems of spectrum shortage in SGCN.

\footnotetext{
* Corresponding Author.

Email Address: salam@numl.edu.pk (S. Alam) https://doi.org/10.21833/ijaas.2017.010.012

2313-626X/C 2017 The Authors. Published by IASE.

This is an open access article under the CC BY-NC-ND license

(http://creativecommons.org/licenses/by-nc-nd/4.0/)
}

The CR is basically an intelligent radio which senses and adapts the parameters (transmission and reception) of a wireless environment in which it operates (Akyildiz et al., 2006). Cognitive radio network (CRN) usually known as a setup, consisting of cognitive devices also known as unlicensed users (secondary users, SUs) which access the unused channels (spectrum holes) vacate by licensed users (primary users, PUs). A CR network architecture (Li et al., 2016) is given in Fig. 1.

All of the technologies that are available to software defined radio (SDR) are employed by the $\mathrm{CR}$ along with the additional competencies of cognition and spectrum sensing (Mitola, 2000). The focus of our work is on spectrum sharing.

Spectrum sharing has three modes of operation; overlay mode: PUs are present and there is collaboration among PUs and SUs, underlay mode: PUs are present and interference threshold is set for SUs and interweave mode: utilize the spectrum holes in the absence of PUs. This work emphasizes on spectrum sharing in Interweave mode where PUs are not considered. CR technology is aimed to avoid interference between PUs and SUs in considered scenario. 


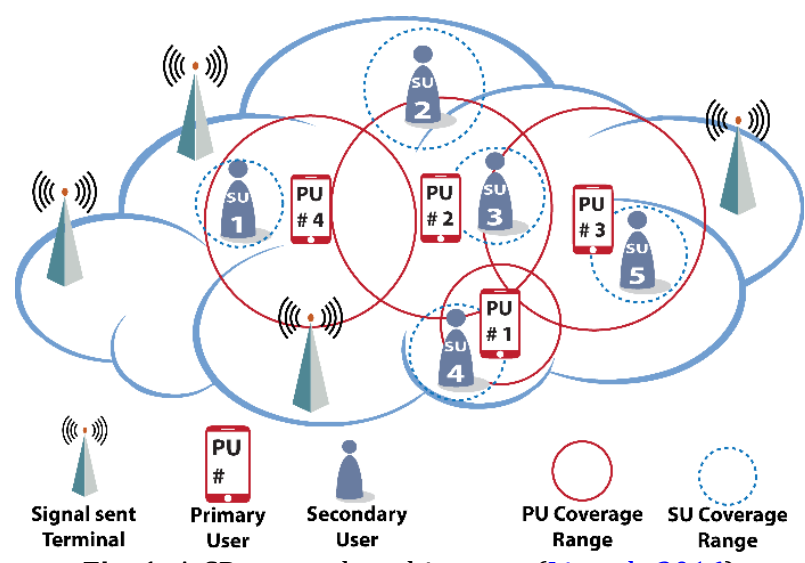

Fig. 1: A CR network architecture (Li et al., 2016)

Smart Grid (SG) is an automatic energy delivering network which enables a two-way communication flow. It is capable of monitoring the entire system, intelligently manages the demand and supply of electricity and to respond to the possible changes

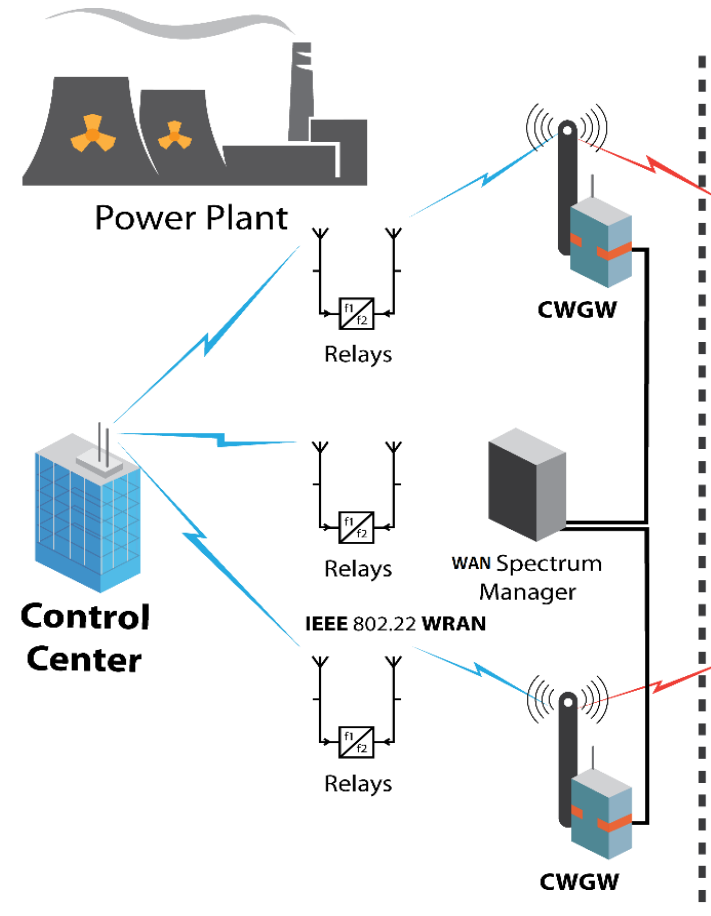

WAN

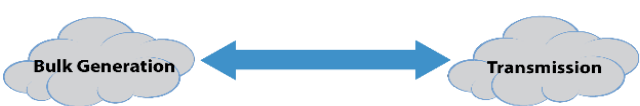

cWGW: Cognitive WAN gateways CNGW: Cognitive NAN gateways HGW: Home Gateways

Fig. 2: Cognitive Radio based Smart Grid network architecture (Alam et al., 2017)
The SG applications generate an enormous amount of data that is needed to be transmitted to (or from) control centers such as meter readings, pricing, service, sensor measurement, multimedia monitoring data, surveillance data, prepayment data, allocation and automation data, firmware updates and program updates (Khan and Faheem, 2014). The number of users, data type, environmental conditions, and cost are the major factors that must be taken into account while selecting communication technology for SG communication. The approximate requirement for data and coverage area of each SGCN communication segment is presented in Fig. 3. that can occur (Alam et al., 2017). Conventional power grids are facing many challenges due to continually rising energy demands (Kim et al., 2014). The SG system is equipped with the two-way communication capabilities for control, monitoring, reliability, and security (Kuzlu et al., 2014). Threelayered network architecture of SGCN is given in Fig. 2. It describes the whole SG communication network as (a) home area network (HAN): user premises (b) neighborhood area network (NAN): distribution and transmission domain, and (c) wide area network (WAN): covering transmission domain to control centre, on the basis of coverage area and data rate required by SG devices. It also depicts the feasible technologies and the nodes/devices that are used in CR operation. The detailed information about HAN, NAN, and WAN can be found in (Alam et al., 2017).
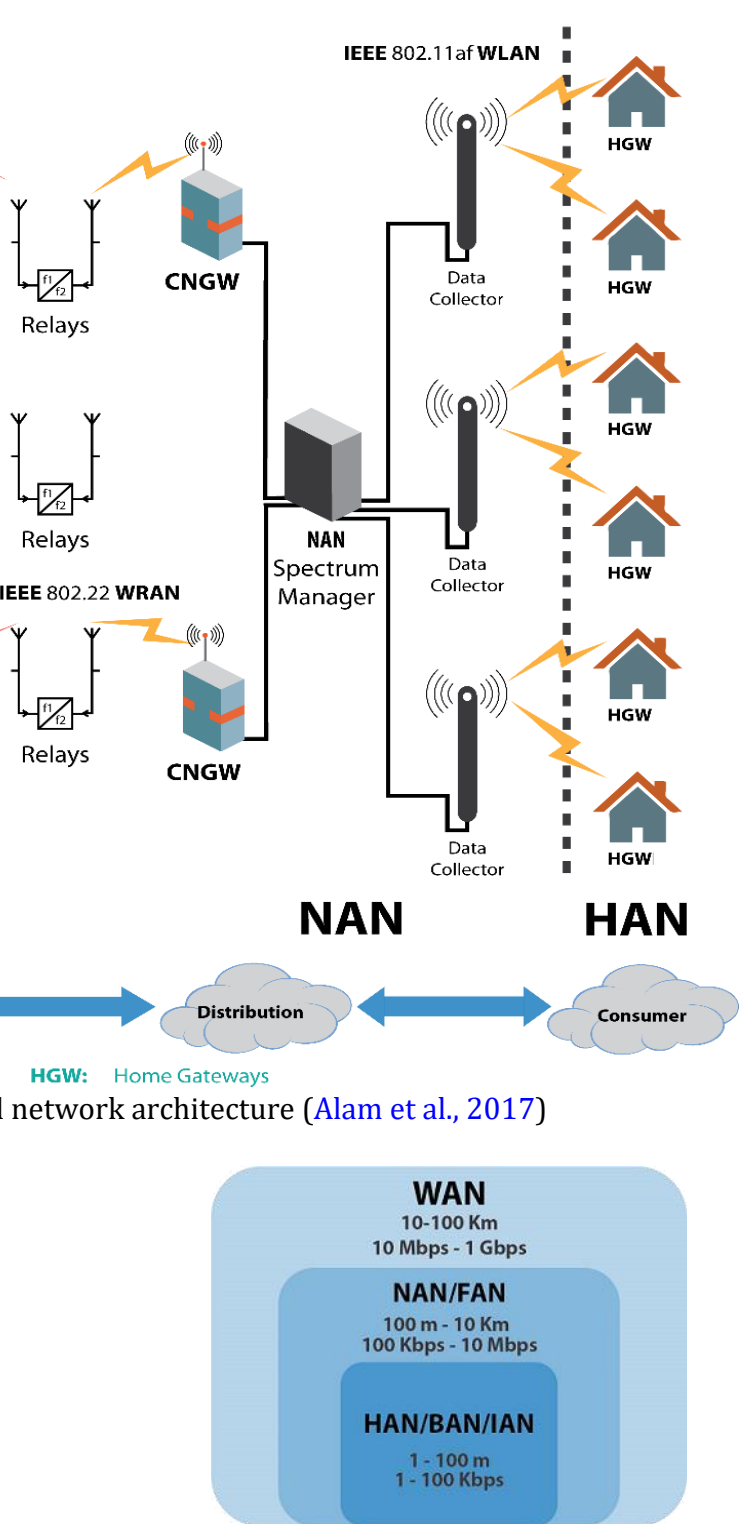

Fig. 3: Approximate coverage and data rate requirements for HAN, NAN, and WAN (Alam et al., 2017)

SGCN is an application area of CR technology, CR is proposed in SGCN to deal with the spectrum 
allocation problem. Cognitive radio (CR) is the motivation to deal with such a huge amount of data and the problems of spectrum shortage; existing literature can be adapted according to the necessities of spectrum resources in SGN.

Fairness is a crucial issue in SGCN to ensure the effectiveness of the system. Each user must be entertained according to its requirements under certain constraints in order to ensure fair distribution of the resources (Khan et al., 2016). In the communication layer of SGN, CR is employed on the basis of fairness by taking into account the overall fairness and user's throughput must be maximized. Spectrum allocation is based on fairness by satisfying all the constraint conditions and it can be analyzed by using Jain's fairness index (section 3, Eq. 1).

Heuristic techniques are proposed in the work to solve this optimization problem and to achieve desirable results. The motivation for using heuristic technique is that it reduces the complexity of the system and reduces computational time, as the time is a critical issue in real time communication. The results show that proposed (heuristic) algorithms achieve the desired results with less computational time and improve the overall spectral efficiency.

The salient features of our proposed methodology, for dynamically allocating the spectrum resources for CR based SGCN, are:

- The considered scenario is based on spectrum sharing in Interweave mode and channel assignment is done in a way to avoid interference with PUs.

- Our work presents an equality based channel allocation i.e. maintaining fairness among users.

- Most of the work has been done by using graph theory but we proposed various heuristic approaches. Graph theory has high computational complexity whereas heuristic algorithms achieve the optimal results with low computational complexity.

- The work mainly focuses on user premises data i.e. AMI metering, billing data etc. that has less time criticality requirement, thus CR is well suited.

In $\mathrm{CR}$, spectrum allocation has been one of the key interest areas for researchers. There are various methods of spectrum allocation that are proposed in the literature to improve the spectral efficiency under required constraints for spectrum allocation in CR. On the other hand, diverse data due to various SG applications to be carried over communication links in SGCN is in massive proportion. It necessitates a fair share of wireless spectrum in an efficient way, thus CR technology is very well suited for this scenario. Fairness is a critical concept in SG, maximizing the user's throughput can cause unfairness in SGN. To solve this problem Byun et al. (2008) and Liu et al. (2008) considers the principle of maximizing the throughput fairness among users, by utilizing some utility functions, e.g. by maximizing the minimum average throughput per SU, using a fairness factor.
Yang et al. (2016) proposed a cognitive smart grid communication networks (CSGCNs) model for Interweave mode, for the fair allocation of spectrum resources by using binary particle swarm optimization. Yu et al. (2010) proposed a new spectrum assignment algorithm for CRN in order to address fairness and throughput problems in underlay mode.

Zhu and Zhou (2008) presented a new model in aiming to stimulate cognition process in a wireless network (in underlay model). Proportional fairness based spectrum allocation algorithm is proposed in (Ge et al., 2010) in which traditional labeling system based channel quantity factor is introduced. It gives better reward in terms of improving user's fairness in underlay model.

In Yang et al. (2009), authors considered the historical allocation information of secondary users and proposed a novel technique to solve the fairness problem by also considering the max sum reward. It improves the results in term of fairness and throughput among users. Fairness based resource allocation in cognitive radio based smart grid network (CRSGN) is presented to solve the aforementioned problems in Interweave mode.

The rest of the paper is organized as Section II describes a review of existing work related to the problem. Section III contains the system and mathematical model of the problem. Heuristic algorithms: Genetic algorithm (GA), Particle swarm optimization (PSO) and Cat swarm optimization (CSO) are discussed in Section IV. Section V explains how heuristic algorithms are applied to the problem under consideration, followed by the simulation and results in section VI. The research article is finally concluded in the last section.

\section{System model and problem formulation}

The system model consists the typical scenario of the CR technology based SGCN, shown in Fig. 3 (Alam et al., 2017). The CR based IEEE 802.22 WRAN (TVWS) and IEEE 802.11af (super Wi-Fi) have been proposed in the literature for NAN and WAN communication. Our proposed solution is feasible for these both communication segments. It is assumed that the NAN and WAN spectrum managers have full knowledge about the spectrum holes. This problem is an actually fair allocation of already sensed spectrum holes among users. Spectrum allocation model comprises of the reward matrix $\mathrm{Ri}$ (the reward that each user will get) assignment matrix $(\mathrm{Ai}, \mathrm{j})$ and reward matrix $(\mathrm{Bi}, \mathrm{j})$.

Jain's fairness index (Eq. 1) is utilized to evaluate the fairness F of CR users, which determine how fairly the resources are allocated.

$F=\frac{\left(\sum_{i=1}^{N} \sum_{j=1}^{M} a_{i, j} b_{i, j}\right)^{2}}{N \sum_{i=1}^{N}\left(\sum_{j=1}^{M} a_{i, j} b_{i, j}\right)^{2}}, \quad M=N \neq 0$

Where $\mathrm{N}$ is a number of cognitive (secondary) users, $\mathrm{M}$ is the total number of spectrums holes, ai,j indicates that the jth channel is given to the ith user 
and bi,j is the benefit of the jth channel assigned to the ith user.

The mathematical model is built on certain constraints, which are interference matrix C, spectrum availability matrix $\mathrm{S}$ and history matrix $\mathrm{H}$. Where $S=\left\{s_{i, j} \mid s_{i, j} \in\{0,1\}\right\}_{N \times M}$ and $s_{i, j}=1$ indicate that the spectrum $\mathrm{j}$ is available to the user i, $C=$ $\left\{c_{i, k, j} \mid c_{i, k, j} \in\{0,1\}\right\}_{N \times M}$ and $c_{i, k, j}=1$ means the interference will occur between $\mathrm{i}$ and $\mathrm{k}$ users if they simultaneously use the jth channel. $H=$ $\left\{h_{T, i} \mid h_{T, i} \in\{0,1\}\right\}_{N \times M}$ where $h_{T, i}$ indicates that how many users get the spectrum and how many are left, in Tth allocation. The fairness among users is measured according to the statistics of the spectrum locations for every single user (Liu et al., 2008). Eq. 2 shows the sum fairness of each user in T allocations.

$F_{i}=\sum_{j=1}^{M} a_{i, j}$

where $\mathrm{T}$ is the number of allocations. Spectrum assignment matrix is $A=\left\{a_{i, j} \mid a_{i, j} \in\{0,1\}\right\}_{N \times M}$ where $a_{i, j}=1$ shows that the jth spectrum is assigned to ith user. Spectrum assignment matrix is assumed to be constant during spectrum assignment.

Fair spectrum resource allocation will guarantee the optimum performance of CSGN system. Each SU's reward which it obtains for a jth channel is (Eq. 3):

$R_{i}=\sum_{j=1}^{M} a_{i, j} b_{i, j}$

where $\mathrm{Ri}$ is per user reward and $\mathrm{M}$ is the number of channels, and the location of these channels varies with each assignment as the environment is dynamic. The max sum reward (MSR) of the CRSGN system is calculated by Eqs. $4 \mathrm{a}$ and $4 \mathrm{~b}$.

$U=\sum_{i=1}^{N} R_{i}$

$U_{o}=\max \sum_{i=1}^{N} \sum_{j=1}^{M} a_{i, j} b_{i, j}$

where Uo is the objective function for the heuristic algorithms applied to our problem to optimize MSR of the system by maintaining fairness.

\section{Algorithms and flowcharts}

This section gives an overview of the heuristic algorithms: genetic algorithm (GA), particle swarm optimization (PSO) and cat swarm optimization (CSO) applied to the problem under consideration. Each algorithm heuristically searches for the optimum solution according to a defined method.

\subsection{Genetic algorithm (GA)}

Genetic algorithms are natural evolution based optimization algorithms which were introduced by Holland (1975). There are two core processes in GA. The 1st one is a selection of individuals to generate the generation and the 2 nd one is the mutation and cross-over for the selection of the final population. An optimum solution for a particular problem is explored by GA in a finite search space (Khan and Ghauri, 2016).

The GA is proposed in this paper to solve the spectrum assignment problem. The flowchart is shown in Fig. 4 and the algorithm is given below:

Step 1: Initialization: In this phase, the initial parameters are initialized for the algorithm and the respective problem.

Step 2: Roulette Wheel Selection: The probabilities of parents (initial +population) are used to generate the offspring in this method. Those with comparatively higher fitness values will get more chances to produce offspring. This is actually the survival of the fittest. Probabilities will be measured according to the following equation (Eq. $5)$, according to the fitness values.

$P_{i}=\sum_{i=1}^{N} \frac{\text { fitness }_{n}}{\text { sum(fitness })}$

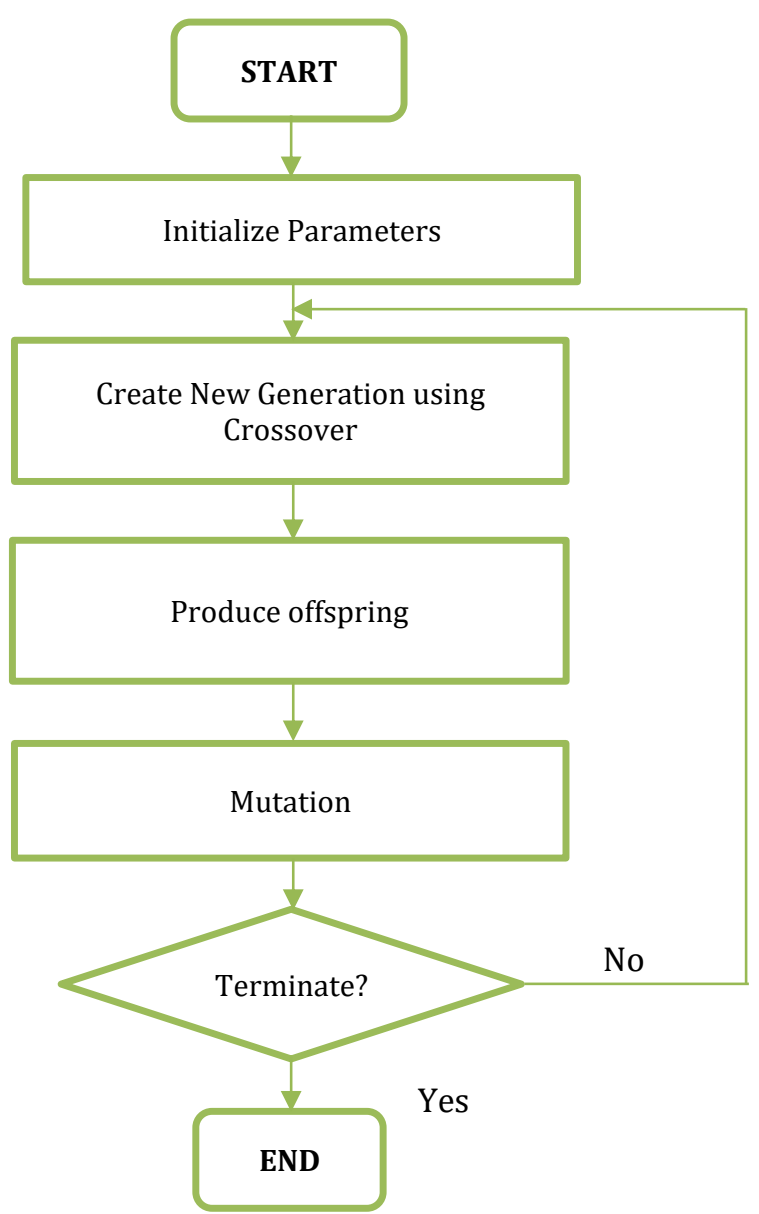

Fig. 4: Flow chart of GA

$\mathrm{Pi}$ and fitness are the probability and fairness of each user respectively.

Step 3: Cross over: Parents and offspring produce next generation by crossover. Genes are randomly shared by parents and offspring to produce a new generation in cross over.

Step 4: Mutation: Mutation is used in GA when the solution is not converging. It improves the selection process by randomly changing some of the 
genes of a population. Sometimes the solution starts diverging in the case of the high mutation rate so, the mutation rate should not be very high.

Step 5: Stoppage Condition: The last step of GA checks if the required results are found. If so, then it will terminate the process. If the solution is not found yet, then GA keeps looking for this iteratively. This process keeps running until the stopping criteria are reached.

\subsection{Particle swarm optimization (PSO)}

PSO, a prodigious evolutionary technique, which was developed by Kennedy and Eberhart (1995). It's basically based on fish schooling and birds flocking's social interaction. The convergence rate of PSO is very fast; this is the reason that it gives better results for fast converging problems as compared to the other heuristic techniques. PSO iteratively updates velocity and position of the particles according to the Eqs. 8 and 9 respectively (Sohail et al., 2017).

The faster convergence rate is the unique quality of PSO due to which it is broadly used in literature to solve the optimization problems. Fig. 5 represents the flowchart and the algorithm consists of following steps.

Step 1: Parameter Initialization: The parameters of problem and algorithm are initialized in this step. The initial random solutions for PSO algorithm is initialized in the form the positions.

Step 2: Fitness Function: In this step, the objective function (given in Eq. 6) is used to calculate the fitness of each random solution. The solutions acquiring the best fitness values are expected to have more survival chances.

Step 3: Velocity and Position Update: Velocity is the vector containing the direction and speed of the particle, while the position is the particle's movement towards the optimal solution. Eqs. 6 and 7 are used for updating the velocities and positions of the swarm, respectively.

$$
\begin{aligned}
& \mathrm{V}^{\prime}(\mathrm{i},:)=\mathrm{w} * \mathrm{~V}(\mathrm{i},:)+\mathrm{c} 1 * \mathrm{r} 1 *(1-\mathrm{r} 2) *[\mathrm{~Pb}(\mathrm{i},:)- \\
& \mathrm{B}(\mathrm{i},:)]+\mathrm{c} 2 * \mathrm{r} 1 * \mathrm{r} 2 *[\mathrm{~Gb}-\mathrm{B}(\mathrm{i},:)]
\end{aligned}
$$

Where V' represents the updated velocity, V indicates the previous velocity. $\mathrm{Pb}$ and $\mathrm{Gb}$ are the local best and global best positions respectively and the $\mathrm{c} 1$ and $\mathrm{c} 2$ are learning factors for local best and global best; $\mathrm{r} 1$ and $\mathrm{r} 2$ are random numbers and $\mathrm{w}$ is the inertia weight.

$S(i, j)=1 /(1+\exp (-V(i, j)))$

where

$\mathrm{S}$ indicates the updated swarm, V represents updated velocities of the individuals.

Step 4: Termination Criteria: The algorithm terminates only if the required solution is found, else it will keep updating the velocities and positions to find the required solution.

\subsection{Cat swarm optimization (CSO)}

The CSO algorithm was proposed by Chu et al. (2006). The algorithm is inspired by the natural behavior of cats. Typically, cats spend maximum time in resting and analyzing their environment for potential prey. This behavior is termed as seeking mode (SM) (Bouzidi and Riffi, 2014). In the second mode, cats move according to their velocities and it is termed as tracing mode (TM).

The mixing ratio (MR) tells that what percentage of cats is going in SM and TM. Flag determines that either the cat is in SM or in TM. The rest of the algorithm is described in the following steps and the algorithm's flow chart is given in Fig. 6.

Step 1: Initialization: In the first step, cats are initialized as the initial solutions. The rest of the parameters like CDC (counts of dimensions to change), SRD (seeking a range of the dimensions), SPC (self-position consideration), SMP (seeking memory pool), flags and MR (mixing ratio) are initialized in this step as well. These parameters are well described in (Chu et al., 2006).

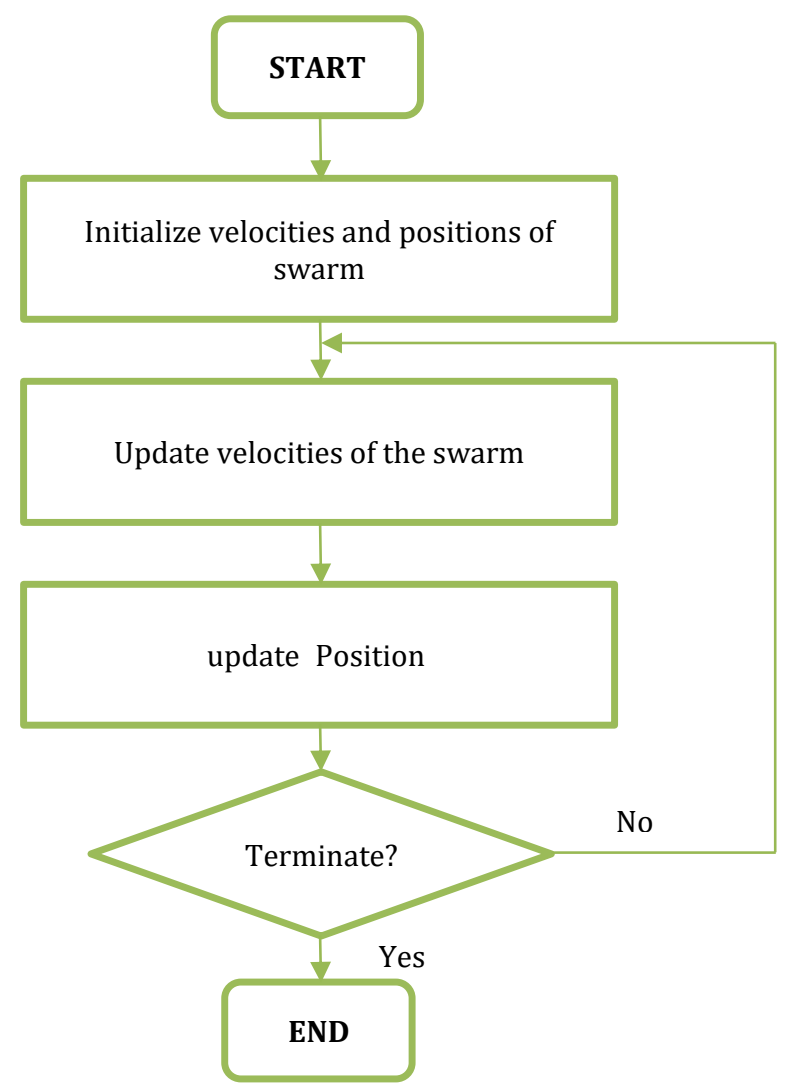

Fig. 5: Flow chart of PSO

Step 2: Is the cat is in SM or TM: The flag of each cat is used to check either the cat will go through SM or TM and how many cats will move in SM and TM, determined by MR.

Step 3: Seeking Mode: In SM mode j copies of the cat are created according to the SMP. Each copy of the cat is updated according to CDC and SRD, randomly. Calculate the fitness of each cat and select a cat randomly. 


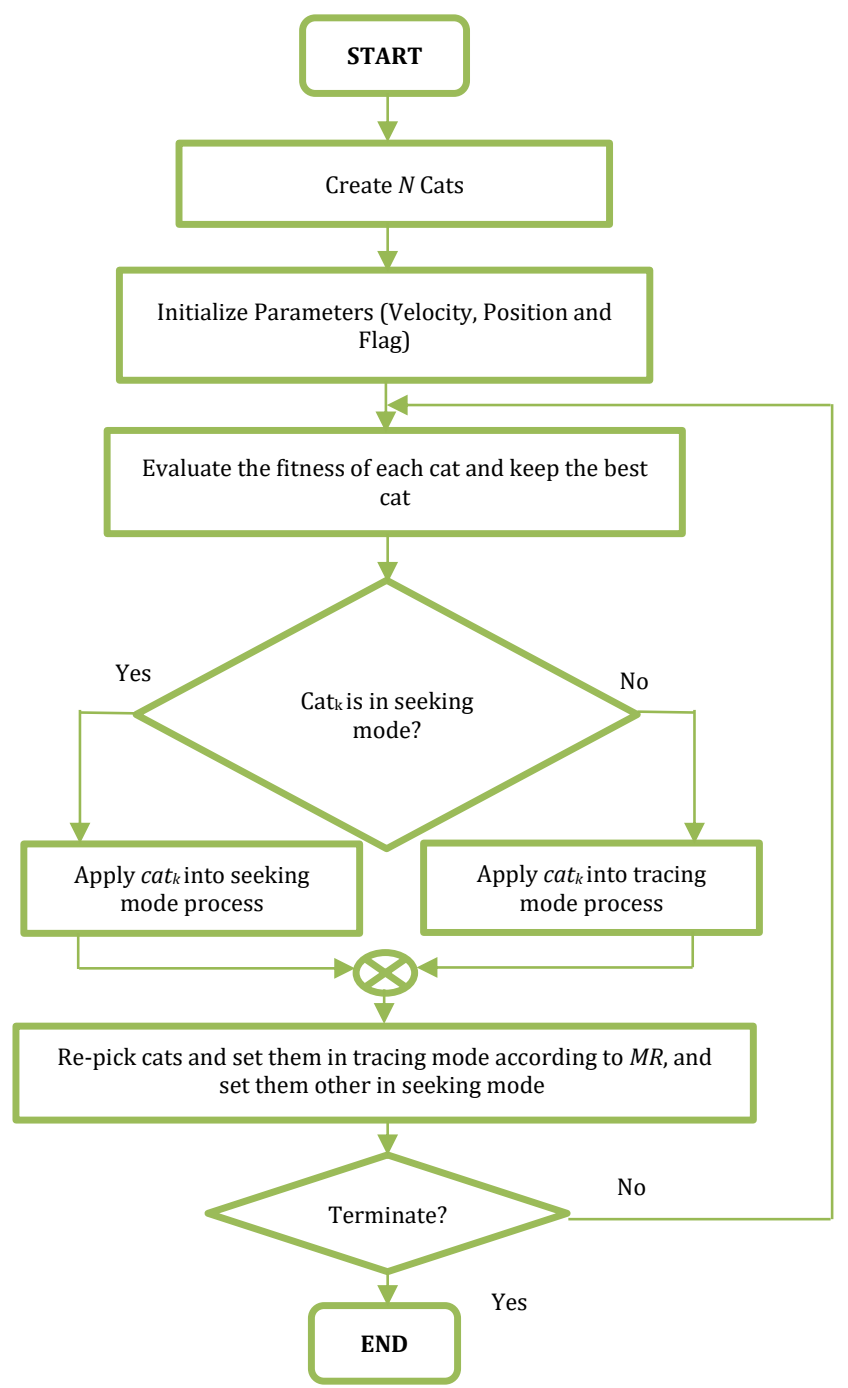

Fig. 6: Flow chart of CSO

Step 4: Tracing Mode: The TM mode determines the next best possible move for each cat. Velocity and position of each cat are updated according to the Eqs. 8 and 9.

$V^{\prime} i, j=V i, j+r 1 c 1(x g b, j-x i, j)$

$x^{\prime} i, j=x i, j+v i, j$

$V^{\prime} i, j$ is the updated velocity and $V i, j$ is the previous velocity, $\mathrm{r} 1$ is the random number while $\mathrm{c} 1$ is the constant factor for global best (Xgb). Whereas $\mathrm{xgb}, \mathrm{j}$ is the cat with the best fitness, $x \mathrm{i}, \mathrm{j}$ is the previous position of the cat and $x^{\prime} i, j$ is the updated position of the cat. The cats, after passing through the seeking mode process are then combined together with the cats of tracing mode.

\section{Algorithm for CRSGCN problem}

Algorithm for CRSGCN is as follows:

Initialize $M, N$, test, $B$, and $m$

for $i=1$ :test

Initialize $S$, Ic and respective algorithm's parameters

for iter=1:iterations

Mapp heuristic solutions to assignment matrix ' $A$ '

Remove co-channel interference

Fairness and MSR calculations
Apply respective algorithm (CSO, GA, PSO, DE or HGAPSO)

Update fairness and MSR

if criteria meet

go to step 16

else go to step 4

end if

end \% iteration loop

repeat

Save user's History

end \% test loop

repeat

return MSR and fairness

Candidate solutions are initialized according to the available channel matrix S. lc is the dimension of each candidate solution (Eq. 10) and it is calculated as:

$l c=\sum_{m=1}^{N} \sum_{n=1}^{M} S_{m, n}$

$\mathrm{M}$ is the availability of channels and $\mathrm{N}$ are the smart grid users (secondary users). The above algorithm shows that how CRSGCN problem is formulated for heuristic algorithms, the algorithm shows the basic steps that is used for the problem to evaluate the optimization problem.

\section{Simulations and results}

For simulation, we assume a noise-free environment where smart grid users are considered as SUs and active licensed users (e.g. licensed analog TV service) using fixed power in a fixed topology where the location of users and spectrum hole remain static during spectrum assignment.

It is supposed that 50 channels $(M=50)$ are randomly distributed among SG users $(\mathrm{N}=20)$ in a service area. Spectrum manager provides the spectrum availability ' $S$ ' according to the necessity of each user, based on channel unused by PUs, which is assumed to be static during the assignment. Channel assignment $A$ is decided on the basis of availability matrix $S$, interference matrix $C$, Reward matrix $B$, and history matrix $\mathrm{H}$ (described in section II). The rest of the parameters are given in Table 1.

Table 1: Simulation parameters

\begin{tabular}{cccc}
\hline Parameters & CSO & PSO & GA \\
\hline c1, c2 & $\ldots \ldots .$. & 1.2 & $\ldots \ldots$. \\
r1, r2 & Random & Random & $\ldots \ldots$. \\
w & 0.7 & 0.7 & $\ldots \ldots$. \\
CDC, SRD SMP, MR & $6 \%, 10 \%, 8,0.3$ & $\ldots \ldots$. & $\ldots \ldots$. \\
Crossover & $\ldots \ldots$. & $\ldots \ldots$. & Single point \\
Mutation & $\ldots \ldots$. & $\ldots \ldots$. & $0.2 \%$ \\
Iterations & 20 & 20 & 20 \\
Population & 10 & 10 & 10 \\
Size & & & \\
\hline
\end{tabular}

Table 2 compares Avg. simulation time for each heuristic algorithm. Comparative analysis of heuristic techniques for fairness and MSR (using Eq. 1 and 4b) are given in Fig. 7 (for fairness) and Table 3 (for MSR).

Fairness among smart grid users in CRSGCN is plotted as channel allocated per SU for no. of allocations in Fig. 7. It is can be observed that min. allocation for GA is 7 where both PSO and CSO have 
the same min. allocation 8. However, CSO is much better for Avg. allocation per SU.

Table 2: Simulation time (i3-4th generation: CPU 1.7 (2) GHz with 4 GB RAM) using MATLAB R2016a

\begin{tabular}{cc}
\hline Heuristic Algorithm & Simulation time (in sec) \\
\hline GA & 7.19 \\
PSO & 7.37 \\
DE & 76.57 \\
CSO & 5.18 \\
Hybrid GA-PSO & 6.97 \\
\hline
\end{tabular}

The results (for MSR) in Table 3 are compared for the four different allocations $(5,10,15$ and20) using each heuristic algorithm. It is observed that percentage MSR decreases with increasing no of allocations. GA has min MSR of $80 \%$ and Avg. MSR of $94 \%$ for 20 allocations. PSO and CSO produce almost similar results for min MSR but for Avg. MSR CSO has an edge over PSO by a small margin of $1 \%$.

The CSO is suggested for the practical implementation for this type of problem as it gives the best results as compared to the other heuristic algorithms. The CSO achieves the overall better performance of the SGCN system as well as it also gives good results for individual users.

\section{Conclusion}

Application of CR technology in SGCN is imperative in SGCN as a major chunk of data due to SG applications is less time critical. CR based communication standards have been widely proposed in the literature. In this research article, we model a typical scenario of SGCN to solve the problem of dynamic spectrum allocation to maximize fairness and MSR among SG users using a heuristic approach, under certain constraints such as channel availability, channel reward, allocation history, and interference among SG users. Comparative analysis of CSO with commonly used GA and PSO techniques is done for fairness and percentage MSR value for different channel allocations. Results have shown (Fig. 7 and Table 3) that CSO outperforms GA in both performance metrics by a large margin. Also, CSO has a slight edge over PSO in Avg. MSR and fairness.

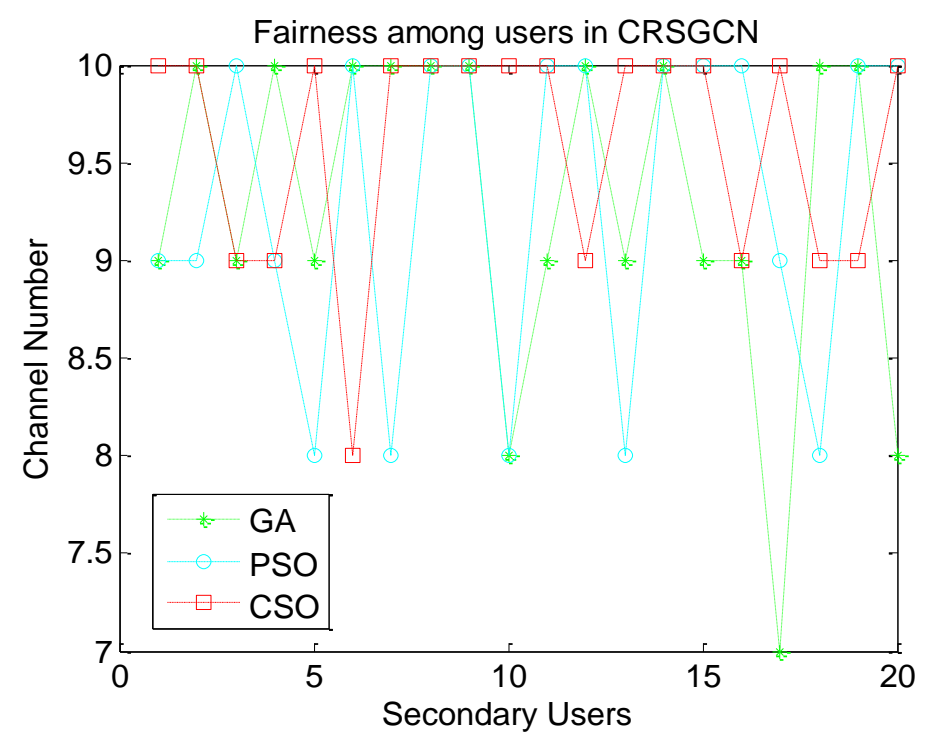

Fig. 7: Fairness of the users in CR based SGCN

Table 3: Simulation results of heuristic algorithms for MSR

\begin{tabular}{cccccccc}
\hline SR \# & No. of Allocations & \multicolumn{2}{c}{ CSO (Percentage) } & \multicolumn{2}{c}{ PSO (Percentage) } & \multicolumn{2}{c}{ GA (Percentage) } \\
\hline & & Min & Avg. & Min & Avg. & Min & Avg. \\
1 & 5 & 90 & 96 & 90 & 95 & 85 & 94 \\
2 & 10 & 90 & 96 & 85 & 92 & 80 & 93 \\
3 & 15 & 85 & 95 & 85 & 93 & 80 & 92 \\
4 & 20 & 85 & 95 & 85 & 94 & 80 & 92 \\
\hline
\end{tabular}

We have assumed fixed topology in a static environment for our model. Effect of mobility of PUs and SUs can be further explored. We have considered interweave mode for our problem, implications of underlay mode can further be studied. New heuristic techniques like Firefly, bat optimization, Grey Wolf optimization etc. can also be explored to further improve the results.

\section{References}

Akyildiz IF, Lee WY, Vuran MC, and Mohanty S (2006). NeXt generation/dynamic spectrum access/cognitive radio wireless networks: A survey. Computer Networks, 50(13): 2127-2159.

Alam S, Sohail MF, Ghauri SA, Qureshi IM, and Aqdas N (2017). Cognitive radio based smart grid communication network. Renewable and Sustainable Energy Reviews, 72: 535-548.

Bouzidi A and Riffi ME (2014). Cat swarm optimization to solve job shop scheduling problem. In the $3^{\text {rd }}$ IEEE International Colloquium in Information Science and Technology, IEEE, Tetouan, Morocco: 202-205. https://doi.org/10.1109/CIST. 2014.7016619

Brown TX (2005). An analysis of unlicensed device operation in licensed broadcast service bands. In the First IEEE International Conference on New Frontiers in Dynamic 
Spectrum Access Networks, IEEE, Baltimore, USA: 11-29. https://doi.org/10.1109/DYSPAN.2005.1542613

Byun SS, Balasingham I, and Liang X (2008). Dynamic spectrum allocation in wireless cognitive sensor networks: Improving fairness and energy efficiency. In the IEEE $68^{\text {th }}$ Vehicular Technology Conference, IEEE, Calgary, Canada: 1-5. https://doi.org/10.1109/VETECF.2008.299

Chu SC, Tsai PW, and Pan JS (2006). Cat swarm optimization. In the Pacific Rim International Conference on Artificial Intelligence, Springer, Berlin, Heidelberg: 854-858. https://doi.org/10.1007/978-3-540-36668-3_94

FCC (2002). Spectrum policy task force report. Federal Communications Commission, Washington, D.C., USA Available online at: sites.nationalacademies.org/cs/groups/ bpasite/documents/webpage/bpa_048826.pdf

Ge Y, Sun J, Shao S, Yang L, and Zhu H (2010). An improved spectrum allocation algorithm based on proportional fairness in Cognitive Radio networks. In the 12th IEEE International Conference on Communication Technology, IEEE, Nanjing, China: 742-745. https://doi.org/10.1109/ICCT.2010.5688612

Holland JH (1975). Adaptation in natural and artificial systems. University of Michigan Press, Ann Arbor, Michigan, USA.

Kennedy J and Eberhart R (1995). Particle swarm optimization. In the IEEE International Conference on Neural Networks, Perth, Australia, 4: 1942-1948. https://doi.org/10.1109/ICNN.1995. 488968

Khan SA and Ghauri SA (2016). Channel estimation using evolutionary computing techniques. International Journal of Technology and Research, 4(1): 16-20.

Khan UU, Dilshad N, Rehmani MH, and Umer T (2016). Fairness in Cognitive Radio Networks: Models, measurement methods, applications, and future research directions. Journal of Network and Computer Applications, 73: 12-26.

Khan ZA and Faheem Y (2014). Cognitive radio sensor networks: Smart communication for smart grids-A case study of Pakistan. Renewable and Sustainable Energy Reviews, 40: 463-474.

Kim SL, Kitsos P, Nekovee M, Yu FR, and Zhang Y (2014). Guest editorial: Smart grid communications systems. IEEE Systems Journal, 8(2): 417-421.
Kuzlu M, Pipattanasomporn M, and Rahman S (2014). Communication network requirements for major smart grid applications in HAN, NAN and WAN. Computer Networks, 67: 74-88.

Li Y, Shen H, and Wang M (2016). Spectrum allocation of cognitive radio based on autonomy evolutionary algorithm. Cybernetics and Information Technologies, 16(4): 87-97.

Liu G, Zhou L, Xiao K, Yu B, Zhou G, Wang B, and Zhu X (2008). Receiver-centric channel assignment model and algorithm in cognitive radio network. In the 4th International Conference on Wireless Communications, Networking and Mobile Computing, IEEE, Dalian, China: 1-4. https://doi.org/10.1109/WiCom.2008.304

Mitola J (2000). Cognitive radio: An integrated agent architecture for software defined radio. Ph.D. Dissertation, Royal Institute of Technology (KTH), Stockholm, Sweden.

Sohail MF, Alam S, Hussain A, Ghauri SA, Sarfraz M, Ahmed MA (2017). Multiuser detection: Comparative analysis of heuristic approach. International Journal of Advanced and Applied Sciences, 4(6): 115- 120.

Yang L, Xie X, and Zheng Y (2009). A Historical-information-based algorithm in dynamic spectrum allocation. In the International Conference on Communication Software and Networks, IEEE, Chengdu Sichuan, China: 731-736. https://doi.org/10.1109/ICCSN.2009.18

Yang S, Wang J, Han Y, and Zhao Q (2016). Dynamic spectrum allocation algorithm based on fairness for smart grid communication networks. In the $35^{\text {th }}$ Chinese Control Conference, IEEE, Chengdu, China: 6873-6877. https://doi.org/10.1109/ChiCC.2016.7554439

Yu L, Liu C, Liu Z, and Hu W (2010). Heuristic spectrum assignment algorithm in distributed cognitive networks. In the $6^{\text {th }}$ International Conference on Wireless Communications Networking and Mobile Computing, IEEE, Chengdu, China: 1-5. https://doi.org/10.1109/WICOM.2010.5600806

Zhu L and Zhou H (2008). A new architecture for cognitive radio networks platform. In the $4^{\text {th }}$ International Conference on Wireless Communications, Networking and Mobile Computing, IEEE, Dalian, China: 1-4. https://doi.org/10.1109/WiCom.2008.327 